\title{
A Model that Forecasts Future Values of Reproductive Success for the Pacific Stock of Splendid Alfonsino
}

\author{
Kazumi Sakuramoto \\ Department of Ocean Science and Technology, Tokyo University of Marine Science and Technology, Tokyo, Japan \\ Email: sakurak@kaiyodai.ac.jp
}

How to cite this paper: Sakuramoto, K. (2021) A Model That Forecasts Future Values of Reproductive Success for the Pacific Stock of Splendid Alfonsino. Open Access Library Journal, 8: e8213.

https://doi.org/10.4236/oalib.1108213

Received: November 22, 2021

Accepted: December 19, 2021

Published: December 22, 2021

Copyright $\odot 2021$ by author(s) and Open Access Library Inc.

This work is licensed under the Creative

Commons Attribution International

License (CC BY 4.0).

http://creativecommons.org/licenses/by/4.0/

\begin{abstract}
A model that forecasts future values of the reproductive success (RPS) for the Pacific stock of Splendid alfonsino (Beryx splendens) using environmental factors is described. The monthly Arctic oscillation index and Pacific decadal oscillation were used as the environmental factors. The RPS from 1999 to 2019 was reproduced and that from 2020 to 2024 was forecasted. The results were as follows: The fitness between the observed and reproduced RPS coincided well. The average RPS from 1999 to 2019 was 0.361 , and that from 2020 to 2024 was 0.370 . Cases in which future values of RPS can be forecast are extremely rare. If future values of RPS can be forecast, the information will contribute to fishery resource management.
\end{abstract}

\section{Subject Areas}

Marine Biology

\section{Keywords}

Reproductive Successes, RPS, Splendid Alfonsino, AO, PDO, Fisheries Management, TAC

\section{Introduction}

In Japan, the total allowable catch (TAC) is determined based on the results of simulations that are conducted assuming the future values of reproductive success (RPS) and by setting several options that determine the reduced rate for the current fishing mortality coefficient, F. The future values of the spawning stock biomass (SSB) and catch are then calculated [1]. In general, however, the average past RPS values are used for the simulation, because the future RPS values are 
not known. However, this procedure has a serious problem when the future RPS value is lower than the average. Estimating the future values of RPS will thus provide useful information for fishery resource management.

Sakuramoto [2] showed that the RPS of 12 stocks around Japan could be reproduced using only environmental factors. The purpose of this paper is to demonstrate a case in which the future values of RPS can be forecast using the method that Sakuramoto showed [2]-[8].

\section{Materials and Methods}

The data for the stocks used in this study were provided by the Fisheries Agency and Fisheries Research Agency of Japan [1]. The environmental factors used in this study were the monthly index of the Arctic oscillation (AO) [9] and the monthly index of the Pacific decadal oscillation (PDO). However, the PDO data that Sakuramoto used [2] were not the newest version. In the present study, the PDO data were replaced by the newest version. [10] The PDO data that were selected here to reproduce the RPS are thus different from those of Sakuramoto [2].

The model that reproduces the RPS herein is essentially the same as that described by Sakuramoto [2].

\section{Results}

\section{Partial Regression Coefficients Used to Reproduce the RPS}

The model used in this paper is as follows:

$$
\begin{aligned}
R P S_{t}= & \exp \left(a_{0}+a_{1} A O_{t-4,7}+a_{2} A O_{t-6,3}+a_{3} P D O_{t-3,3}+a_{4} P D O_{t-3,4}\right. \\
& \left.+a_{5} P D O_{t-3,7}+a_{6} P D O_{t-5,12}\right)
\end{aligned}
$$

where $R P S_{t}$ denotes the reproductive success in year $t, A O_{t-i, m}$ represents the $\mathrm{AO}$ in month $m$ of year $t-i$, and $P D O_{t-k, m}$ is the PDO in month $m$ of year $t-k . a_{i}$ are the partial regression coefficients, the estimated values of which are: $a_{0}=$ $-1.01640, a_{1}=0.37528, a_{2}=0.14709, a_{3}=0.15714, a_{4}=-0.31603, a_{5}=-0.10595$, and $a_{6}=-0.08927$.

Figure 1 shows the observed and reproduced RPS values (see Appendix). The entire tendency is well reproduced. Further, the RPS abruptly decreased in 2018 and 2019, and these sudden and rapid decreases were well reproduced with the model. This indicates that the model proposed here is reasonable.

At this time (September 2021), the values of RPS and SSB are published from 1999 to 2019 [1]. As mentioned above, the environmental data up to July in year $t-3$ are necessary to calculate the RPS in year $t$. As of September 2021, the AO and PDO data are available up until August 2021 [9] [10]. Therefore, the future values of RPS until 2024 can be reproduced.

The top of Figure 2 shows the forecasted RPS from 2020 to 2024. The RPS value recovered in 2020 and became significantly high in 2021 . The average RPS value for the period from 2020 to 2024 is 0.370 , which is not significantly different from 0.361, which is the average RPS from 1999 to 2019. 


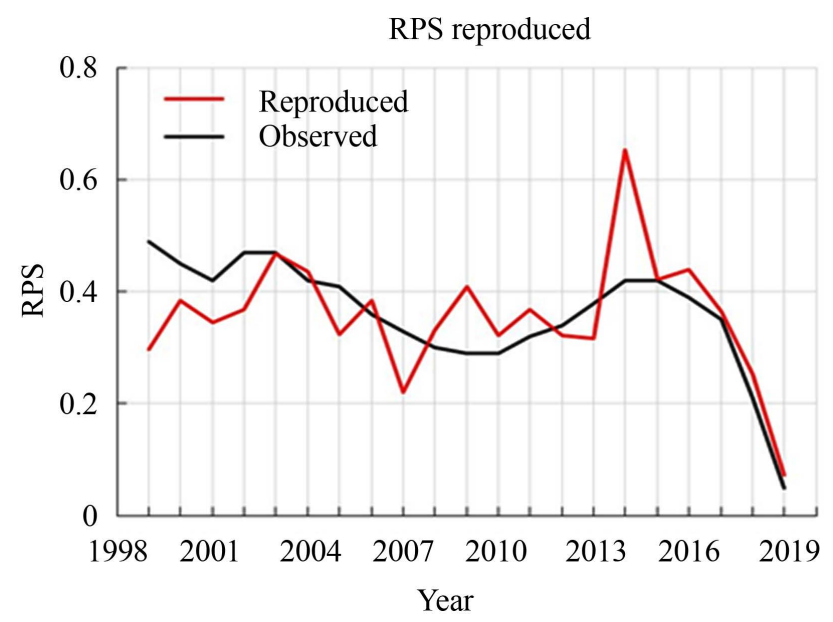

Figure 1. RPS observed (black) and reproduced (red).

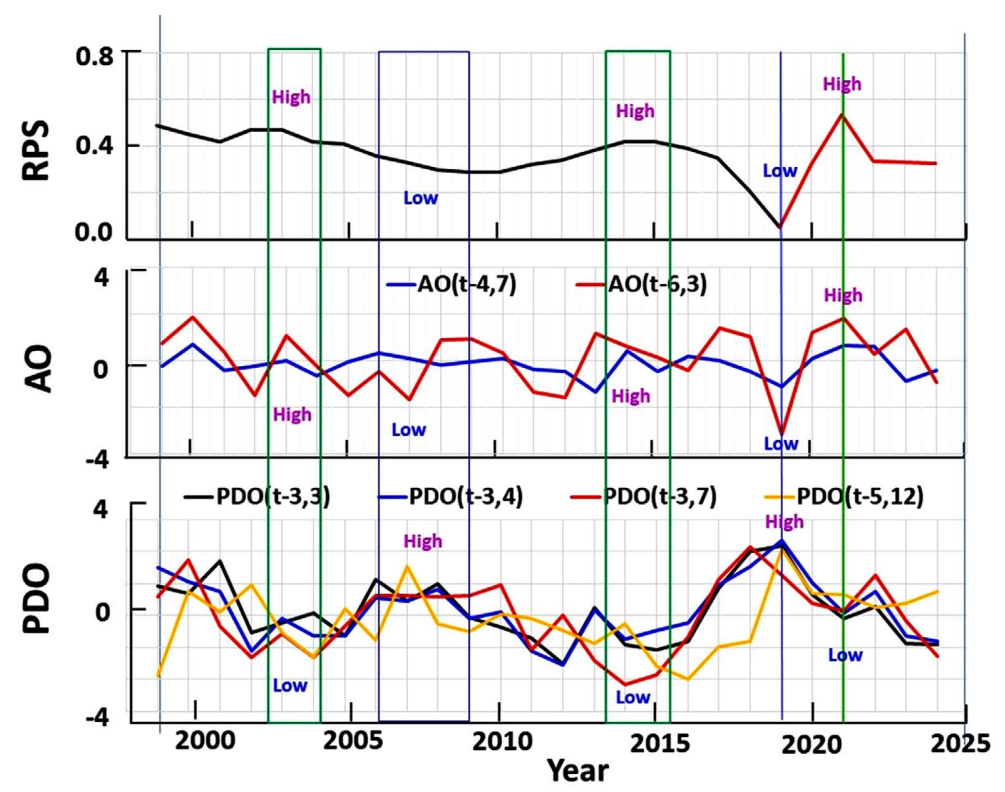

Figure 2. Relationship between the trajectories of RPS and those of environmental factors.

The middle of Figure 2 shows the trajectories of the AO in July of year $t-4$ and March of year $t-6$, that were used to reproduce the RPS. The bottom of Figure 2 shows the trajectories of PDO in March, April and July of year $t-3$, and December of year $t-5$ that were used to reproduce the RPS.

The RPS was high around 2003 and 2014, and it was low around 2008 and 2019. The AO was high around 2003 and 2014 and low around 2007 and 2019. The PDO was low around 2003 and 2014 and high around 2007 and 2019. It can thus be said that the RPS has a positive correlation with the AO and a negative correlation with the PDO. Regarding future values of RPS, the AO values in September 2017 and March 2015 (which are plotted at 2021 in the x-axis of the middle of Figure 2) were both high, and the PDO data in March, April and July of 2018, and December 2016 (which are plotted at 2021 in the $\mathrm{x}$-axis of the bot- 
tom of Figure 2) were low. This is the reason why the forecasted value of RPS in 2021 was evaluated as high.

\section{Discussion}

The results of this study demonstrate that the RPS can be reproduced using only the AO and PDO indexes by month. As shown in Figure 2, the RPS has a positive correlation with the $\mathrm{AO}$ and a negative correlation with the PDO. The case of the Pacific stock of Splendid alfonsino is a special case because their life span is very long; their maximum age is 26 years, and their maturity age is also high. Approximately $50 \%$ of 4 -year-old Splendid alfonsino are mature, and $100 \%$ of the $\geq 5$-year-old and older fish are mature. The approach described herein can therefore not be adopted to many other stocks of fish in which the life span and maturity age are short. However, if the target species has similar features, it is worthwhile to try to use this approach to forecast future values of RPS to reduce the uncertainty of the species' management.

\section{Acknowledgements}

I thank anonymous reviewers for their useful comments that improved this manuscript.

\section{Conflicts of Interest}

The author declares no conflicts of interest.

\section{References}

[1] Fisheries Agency and Fisheries Research and Education Agency of Japan (2020) http://www.abchan.fra.go.jp/digests2020/details/202037.pdf

[2] Sakuramoto, K. (2021) Reproductive Success in Fish Stocks Can Be Reproduced by Environmental Factors Alone. Open Access Library Journal, 8, e7636. https://doi.org/10.4236/oalib.1107636

[3] Sakuramoto, K. (2015) A Stock-Recruitment Relationship Applicable to Pacific Bluefin Tuna and the Pacific Stock of Japanese Sardine. American Journal of Climate Change, 4, 446-460. https://doi.org/10.4236/ajcc.2015.45036

[4] Sakuramoto, K. (2013) A Recruitment Forecasting Model for the Pacific Stock of the Japanese Sardine (Sardinops melanostictus) that Does Not Assume DensityDependent Effects. Agricultural Sciences, 4, 1-8. https://doi.org/10.4236/as.2013.46A001

[5] Sakuramoto, K. (2014) A Common Concept of Population Dynamics Applicable to Both Thrips imaginis (Thysanoptera) and the Pacific Stock of the Japanese Sardine (Sardinops melanostictus). Fisheries and Aquaculture Journal, 4, 140-151. https://doi.org/10.4172/2150-3508.1000085

[6] Sakuramoto, K. (2016) Case Study: A Simulation Model of the Spawning Stock Biomass of Pacific Bluefin Tuna and Evaluation of Fisheries Regulations. American Journal of Climate Change, 5, 245-260. https://doi.org/10.4236/ajcc.2016.52021

[7] Sakuramoto, K. (2016) Density-Dependent Effect Occurs Regardless of Density. Open Access Library Journal, 3, e3112. https://doi.org/10.4236/oalib.1103112 
[8] Sakuramoto, K. (2015) Illusion of a Density-Dependent Effect in Biology. Agricultural Sciences, 6, 479-488. https://doi.org/10.4236/as.2015.65047

[9] https://www.cpc.ncep.noaa.gov/products/precip/CWlink/daily ao index/monthly.a o.index.b50.current.ascii

[10] https://www.data.jma.go.jp/gmd/kaiyou/data/db/climate/pdo/pdo.txt 


\section{Appendix}

Model used is:

$$
\begin{aligned}
R P S_{t}= & \exp \left(a_{0}+a_{1} * A O_{t-4,7}+a_{2} * A O_{t-6,3}+a_{3} * P D O_{t-3,3}+a_{4} * P D O_{t-3,4}\right. \\
& \left.+a_{5} * P D O_{t-3,7}+a_{6} * P D O_{t-5,12}\right)
\end{aligned}
$$

\begin{tabular}{|c|c|c|c|c|c|c|c|c|}
\hline \multirow{2}{*}{ year } & AO & $\mathrm{AO}$ & PDO & PDO & PDO & PDO & RPS & RPS \\
\hline & Jul & Mar & Mar & Apr & Jul & Dec & Obs. & Rep. \\
\hline 1993 & -0.5110 & 0.7640 & 0.6692 & 1.2264 & 1.6171 & 0.6966 & & \\
\hline 1994 & 0.3510 & 1.8810 & 0.6426 & 0.8729 & -0.1740 & -1.8868 & & \\
\hline 1995 & -0.2170 & 0.3930 & 0.7950 & 0.7486 & 1.4680 & 0.7715 & & \\
\hline 1996 & 0.7150 & -1.4830 & 0.9253 & 1.5036 & 0.6022 & 0.1159 & & \\
\hline 1997 & -0.4310 & 1.0910 & 0.6871 & 1.0747 & 1.7264 & 0.9542 & & \\
\hline 1998 & -0.2120 & -0.2540 & 1.7207 & 0.7457 & -0.3158 & -0.5223 & & \\
\hline 1999 & -0.0020 & -1.4920 & -0.5259 & -1.0965 & -1.3149 & -1.3216 & 0.49 & 0.30 \\
\hline 2000 & -0.6490 & -0.4510 & -0.2172 & -0.0793 & -0.5711 & 0.2154 & 0.45 & 0.38 \\
\hline 2001 & -0.0310 & -1.6870 & 0.0923 & -0.6283 & -1.2922 & -0.7812 & 0.42 & 0.35 \\
\hline 2002 & 0.3280 & 0.9020 & -0.6251 & -0.6400 & -0.3211 & 1.5521 & 0.47 & 0.37 \\
\hline 2003 & 0.0750 & 0.9330 & 1.1233 & 0.5578 & 0.6213 & -0.2561 & 0.47 & 0.47 \\
\hline 2004 & -0.2010 & 0.3180 & 0.4634 & 0.4619 & 0.6246 & -0.4884 & 0.42 & 0.44 \\
\hline 2005 & -0.0300 & -1.3480 & 0.9934 & 0.8321 & 0.5775 & 0.0574 & 0.41 & 0.32 \\
\hline 2006 & 0.1030 & -1.6040 & -0.0463 & -0.0884 & 0.6315 & -0.0744 & 0.36 & 0.38 \\
\hline 2007 & -0.3970 & 1.1820 & -0.3529 & 0.1201 & 0.9694 & -0.4671 & 0.33 & 0.22 \\
\hline 2008 & -0.4800 & 0.5860 & -0.6805 & -1.1173 & -1.0584 & -0.8644 & 0.3 & 0.33 \\
\hline 2009 & -1.3560 & 0.1210 & -1.4949 & -1.5554 & 0.0090 & -0.2541 & 0.29 & 0.41 \\
\hline 2010 & 0.4350 & -0.4320 & 0.2453 & 0.1858 & -1.3971 & -1.5909 & 0.29 & 0.32 \\
\hline 2011 & -0.4720 & 1.4240 & -0.8965 & -0.7402 & -2.1449 & -1.9915 & 0.32 & 0.37 \\
\hline 2012 & 0.1680 & 1.0370 & -1.0674 & -0.4479 & -1.8592 & -0.9832 & 0.34 & 0.32 \\
\hline 2013 & -0.0110 & -3.1850 & -0.7881 & -0.2219 & -0.6705 & -0.7854 & 0.38 & 0.32 \\
\hline 2014 & -0.4890 & 1.2060 & 0.8634 & 0.9515 & 1.1208 & 2.0744 & 0.42 & 0.65 \\
\hline 2015 & -1.1080 & 1.8370 & 2.0246 & 1.5341 & 2.1565 & 0.6906 & 0.42 & 0.42 \\
\hline 2016 & 0.0850 & 0.2800 & 2.1924 & 2.3471 & 1.2806 & 0.6502 & 0.39 & 0.44 \\
\hline 2017 & 0.6340 & 1.3650 & 0.6490 & 1.0200 & 0.3750 & 0.2520 & 0.35 & 0.37 \\
\hline 2018 & 0.6120 & -0.9410 & -0.1000 & 0.0853 & 0.1389 & 0.3899 & 0.21 & 0.25 \\
\hline 2019 & -0.8900 & 2.1160 & 0.3011 & 0.7590 & 1.2764 & 0.7549 & 0.05 & 0.07 \\
\hline 2020 & -0.4120 & 2.6410 & -0.8551 & -0.6237 & -0.1704 & -0.2030 & & 0.32 \\
\hline 2021 & 0.6250 & 2.1090 & -0.8914 & -0.8143 & -1.2792 & & & 0.54 \\
\hline 2022 & & & & & & & & 0.33 \\
\hline 2023 & & & & & & & & 0.33 \\
\hline 2024 & & & & & & & & 0.32 \\
\hline
\end{tabular}

$\begin{array}{ccccccc}a_{0} & a_{1} & a_{2} & a_{3} & a_{4} & a_{5} & a_{6} \\ -1.01640 & 0.37528 & 0.14709 & 0.15714 & -0.31603 & -0.10595 & -0.08927\end{array}$

